\title{
LANGUES ET LITTÉRATURES DE LA «VIEILLE EUROPE » DANS LES UNIVERSITÉS AMÉRICAINES : UN ÉTAT DES LIEUX
}

Le libéralisme ne se conçoit guère sans une critique du libéralisme ${ }^{1}$.

Parler de l'enseignement des langues et littératures étrangères aux États-Unis nécessite qu'on établisse avant tout de claires distinctions entre les différents établissements supérieurs qui accueillent ce type de programmes au niveau universitaire. D'une institution à l'autre, la situation est souvent très variable et ce qui vaut pour l'une ne vaut pas forcément pour toutes les autres. Étant donné l'absence d'un système universitaire national, comme c'est le cas dans la plupart des pays européens, qui dépendrait de l'état fédéral sous la responsabilité d'un ministère de l'éducation, la responsabilité de gestion des établissements publics d'enseignement supérieur incombe le plus souvent, quand ces établissements ne sont pas privés, aux autorités locales, c'est-à-dire aux états (ce sont les state universities ou universités d'état) ainsi qu'aux comtés et aux villes (on parle alors de community colleges). Les premiers, de grande taille, proposent des diplômes pouvant mener jusqu'au doctorat alors que les seconds, de taille beaucoup plus réduite, se limitent aux deux premières années du premier cycle (les étudiants doivent ensuite rejoindre un autre établissement pour obtenir un Bachelor of Arts, l'équivalent de notre licence). Cette répartition entre universités et community colleges publics se double d'une seconde distinction entre les établissements appartenant au secteur

${ }^{1}$ A. Thibaudet, « Propos sur la critique », [dans :] Réflexions sur la politique, Robert Laffont, Collection « Bouquins », Paris 2007, p. 1211. 
public d'une part, que nous venons d'évoquer, et les institutions privées, d'autre part, qui elles-mêmes se divisent entre universités et four-year liberal arts colleges (souvent désignés par colleges), selon les mêmes critères que ceux indiqués plus haut. Le recrutement des étudiants et des enseignants, les frais d'inscription, les bourses, les salaires des enseignants et du personnel administratif, les conditions de vie et de travail sur le campus et les opportunités en terme d'avancement et d'aide à la recherche, pour ne citer que ces aspects-là, varient énormément d'un secteur à l'autre, moins en fait entre les universités publiques et les community colleges, comme on aurait tendance à le penser, qu'entre les établissements du secteur public et ceux du secteur privé, ce qui rend toute tentative de comparaison sans doute futile et dénuée de pertinence.

Si les community colleges publics continuent à proposer un enseignement (limité certes en ce qui concerne la variété des disciplines offertes et des cours proposés et le niveau de ces derniers) à un tarif encore abordable pour l'étudiant américain moyen, on observe que depuis les années 80 le fonctionnement des universités publiques a tendance à s'éloigner de celui des autres établissements publics pour se rapprocher davantage de celui des universités privées. Sous l'effet de la politique de désengagement des états vis-à-vis de l'éducation, afin de maintenir l'équilibre des budgets et un niveau de taxation des contribuables assez bas par rapport à ce qui est le cas dans la plupart des pays européens, les universités d'état ont été contraintes de se tourner progressivement vers des formes de financement d'origine privée (fondations, laboratoires, entreprises, mécénat, etc.) afin de compenser la baisse des dotations venant des états, si bien que de très nombreuses universités publiques américaines n'ont désormais de public que le nom puisque la majorité de leurs revenus proviennent non plus des états mais des dons privés et des frais d'inscription qui, suivant le modèle des universités et des colleges du secteur privé, n'ont parallèlement cessé d'augmenter ${ }^{2}$. La crise des prêts hypothécaires à risque (subprimes mortgage crisis), qui a touché le système bancaire américain de plein fouet à partir de l'été 2007, a encore aggravé la situation des universités publiques. Citons, en guise d'exemple, un chiffre qui permettra de mieux comprendre la rapidité et la force de cette évolution : sur les trente-cinq dernières années (c'est-à-dire depuis la première élection de Ronald Reagan à la présidence américaine et le début de la politique de dérégularisation et du démantèlement systématique par les Républicains des services publics au profit du privé), les frais

2 En 2012, un rapport conjoint du Department of Treasury et du Department of Education indiquait que « in 1987, four-year, public institutions derived 60 percent of their total revenue from state government support and 20 percent from student tuition payments. By 2009, the composition had shifted substantially — state government funding constituted only 40 percent of revenue while tuition payments constituted another 40 percent. Put another way, tuition, as a share of college revenue, doubled while state government support fell by approximately 33 percent » (The Economic of Higher Education, décembre 2012, p. 3, <https://www.treasury.gov/connect/blog/Documents/20121212_Economics\%20of\%20Higher\%20Ed_vFINAL.pdf $>$ ). 
d'inscription des universités publiques américaines ont quasiment plus que doublé dans le public, passant à une moyenne qui avoisinait les dix mille dollars par an par étudiant en $2013^{3}$. Bien entendu ce chiffre demeure relativement bas en comparaison des frais d'inscription de certaines universités privées, qui peuvent aller au-delà de cinquante mille dollars par an, il est néanmoins très élevé par rapport aux tarifs pratiqués par la plupart des universités publiques européennes (en 2018, les frais d'inscription à Columbia University s'élèvent à \$57,208).

Bien entendu, ces bouleversements des modes de financement et de fonctionnement du système universitaire américain ont eu un impact particulièrement important sur les établissements publics qui, pendant très longtemps, étaient les plus tributaires des dotations des états. Il va sans dire que ces bouleversements ont occasionné des changements structurels qui touchent, pour ce qui nous concerne ici, les facultés de langues et littératures romanes (aux États-Unis on parlera le plus souvent de departments plutôt que de facultés), comme la plupart des autres facultés représentant les sciences humaines. Ces changements se sont notamment traduits par des refontes structurelles ainsi que par des modifications au niveau des appellations. Traditionnellement, selon la taille de l'université et son histoire personnelle, les facultés de langues ont été réunies dans les universités américaines sous le nom de Department of Foreign Languages and Literatures ou Department of Modern Languages and Literatures, un peu moins souvent sous celui de Department of Romance Languages and Literatures (comme c'est encore le cas pour les universités d'Harvard, de Chicago et d'Oregon, par exemple). Notons que le terme "literature » n'apparaît pas toujours dans l'intitulé de la faculté même si l'enseignement de cours de littérature y est bien assuré. Pour des raisons qui tiennent à la fois à l'importance du nombre des enseignants, chercheurs et étudiants dans une seule et même faculté, à l'histoire et à la tradition locale et enfin au prestige lié à telle ou telles langue et littérature, certaines universités ont préféré maintenir des facultés indépendantes (Department of French, par exemple, à l'Université de Californie à Berkeley ou à Columbia University), ou bien ont réuni l'étude de deux langues au sein d'une seule faculté comme c'est le cas du Department of French and Italian (à l'Ohio State University et à l'Université d'Arizona, par exemple) ou Department of Spanish and Portuguese (à l'Université du Colorado à Boulder et à l'Université du Texas à Austin, par exemple). Il est évident que, sous les effets des restrictions budgétaires de ces dernières décennies, la tendance n'est plus à l'éparpillement des unités d'enseignement et de recherche mais plutôt à un rassemblement permettant à ces unités de partager les ressources et de réaliser des économies sur les dépenses liées à leur fonctionnement. Même une université privée et prestigieuse comme la Johns Hopkins University à Baltimore,

${ }^{3}$ Toujours selon The Economic of Higher Education : « Average posted in-state tuition for four-year, public institutions more than doubled between 1991 and 2013, from $\$ 3,350$ to $\$ 8,660$. Average posted tuition at four-year, private non-profit universities rose 57 percent between 1991 and 2013 , from $\$ 16,410$ to $\$ 29,060$ ». 
par exemple, a vu, dès 1999, son Department of French se couler au sein d'une entité plus large, intitulée Department of Romance Languages, accueillant également l'ex Department of Hispanic and Italian Studies. Dans certains cas, des universités n'ont réussi à maintenir la multiplicité des langues qu'en les rassemblant sous l'égide de structures plus vastes les englobant comme c'est le cas, en 2008 à Arizona State University, de la création d'une School of International Letters and Cultures recouvrant vingt-deux langues, à l'intérieur de laquelle se retrouvent non pas des departments mais des facultés (le nom est repris aux universités européennes) regroupées selon les modèles en vigueur ailleurs (Faculty of French and Italian, Faculty of Spanish and Portuguese, Faculty of East and Southeast Asian Studies, etc.). On peut donc établir que l'histoire récente de la nomenclature des departments des universités américaines résulte en grande partie des bouleversements économiques observés aux États-Unis depuis les années 80 et qu'elle reflète bien au niveau structurel le nouvel ordre économique mondial.

L'économie n'est bien sûr pas seule responsable des changements intervenus ces trente dernières années dans le système universitaire américain et celui de l'enseignement des langues et littératures romanes. L'évolution politique du monde occidental et le processus de globalisation, et les conséquences qu'ils ont entraînées plus particulièrement sur la mentalité des Américains et les mutations de la société américaine, ont profondément participé à la transformation des universités en général et des departments de langues et littératures étrangères en particulier. Les processus de la décolonisation (du Vietnam à l'Algérie, par exemple) et les mouvements en faveur des droits des personnes de couleur, de la femme et des homosexuels, et ce que l'on a nommé la libération sexuelle en général (ce qu'on appelle les civic rights aux États-Unis), ont eu un impact très fort dès les années 70 sur le monde académique américain auquel appartenaient déjà nombre d'activistes. Mais il aura fallu encore une génération au moins, c'est-à-dire jusqu'au début des années 80 , pour que les acteurs et les idées de cette « révolution culturelle » s'imposent au sein des diverses instances des universités américaines et notamment dans les departments de langues étrangères et/ou romanes ${ }^{4}$. La question de la représentation des minorités (raciales, sexuelles, etc.) et le discours contre la doxa se sont donc imposés lentement et tout naturellement dans les milieux universitaires et dans le cursus des facultés comme le versant académique des bouleversements géopolitiques et sociaux intervenus dans le monde à partir de la fin de la seconde guerre mondiale

${ }^{4}$ On a du mal à se rendre compte, en 2017, à quel point les intellectuels français en particulier (la triade formée par Derrida, Foucault et Barthes, notamment) jouissaient d'un statut et d'une réputation inégalés dans le monde académique nord-américain des années 70 et 80 , et ce bien au-delà des facultés de français et/ou de langues romanes. Il n'est pas un département d'anglais ou de littérature comparée aux États-Unis qui n'ait compté, tout au long de ces années, un ou plusieurs spécialistes de ce que l'université américaine désigne sous le nom de «French Thought ». C'est aussi l'époque où René Girard et Michel Serres quittaient la France pour accepter des postes dans les universités américaines les plus prestigieuses. 
et de la décolonisation. Le regard empreint de méfiance vis-à-vis de ce qui était alors considéré comme le canon littéraire, et la remise en cause parfois agressive et même outrancière de ce dernier, sont à l'origine des "post-colonial studies ", « cultural studies 》, «feminist studies 》, « gender studies », « gay studies » et autres « queer studies » qui constituent désormais la norme - une nouvelle doxa si l'on veut - du discours universitaire américain contemporain dans de très nombreuses universités dont les plus prestigieuses et influentes avec toutes les dérives que cela implique. Cela s'est aussi traduit par un profond renouvellement du choix des auteurs et des textes étudiés dans les programmes des cours de littérature au point où tout enseignant de littérature française moderne aurait aujourd'hui des scrupules, vis-à-vis de ses collègues en tout cas, à demander à ses étudiants de commenter des romans de Henry de Montherlant ou de Julien Green, si la création des programmes ne demeurait encore - rare privilège spécifique à l'Amérique — le produit d'un choix fort heureusement individuel pour les enseignants des universités américaines.

Le bouleversement de ce que l'on appelle «the canon » marque aussi d'une certaine manière les limites, voire la fin, de la suprématie de la pensée et de la littérature européennes en général - et françaises en particulier — aux États-Unis, au profit d'un discours critique spécifique aux universitaires américains voire même anglo-saxons, pour qui la littérature n'est plus la référence absolue mais seulement l'un des supports utilisés parmi tant d'autres, quand bien même ce discours critique prend paradoxalement le plus souvent son origine et une grande partie de ses fondements dans les œuvres d'intellectuels européens ou d'origine et/ou de formation européennes tels qu'Adorno, Althusser, Barthes, Benjamin, Bourdieu, Cixous, Deleuze, Derrida, Eliade, Fanon, Foucault, Habermas, Levinas, LéviStrauss et Todorov, pour n'évoquer que les noms les plus souvent cités par les critiques nord-américains ces trente ou quarante dernières années ${ }^{5}$. Concrètement, cet éloignement - prise de distance ou émancipation, selon la façon dont on l'interprète - vis-à-vis de la littérature comme référent de base et des grandes figures de la culture européenne a pu se traduire ici et là par la reformulation du nom de certains departments de langue et littérature romanes de manière à refléter plus clairement la nouvelle identité des chercheurs et la nature de leur enseignement. Ainsi, en 2015, le Department of Romance Languages and Literatures de l'Université de Caroline du Nord à Chapel Hill abandonnait-il son appellation plus que centenaire au profit du titre de Department of Romance Studies. Pour Federico Luisetti, son directeur, ce titre « reflète le nouveau visage des études des langues,

${ }^{5}$ Notons cependant, en guise d'exemple, que c'est paradoxalement grâce au développement des « gay studies » puis des "queer studies » qu'un auteur comme André Gide a pu se maintenir dans le discours critique américain contemporain et continuer à attirer l'attention des étudiants sur celui qui fut un temps (désormais bien révolu, il faut bien le reconnaître) considéré, selon le mot d'André Rouveyre, comme le « contemporain capital ». Voir F. Canovas, « D'est en ouest : itinéraire de la critique gidienne aux États-Unis de 1951 à nos jours », [dans :] Actualités d'André Gide, M. Sagaert et P. Schnyder (dir.), Honoré Champion, Paris 2012, pp. 197-214. 
littératures et cultures et complémente les récents changements intervenus dans la structure administrative de la faculté et de ses programmes de master et de doctorat ainsi que dans la culture de l'université ».

Ce revirement suit une tendance nationale observée dans d'autres facultés d'études romanes, dans des établissements comme les universités de Cornell et de Duke, qui ont tenté de mettre un terme aux barrières traditionnelles qu'elles soient d'ordre méthodologique ou d'ordre national pour insister sur la recherche et la publication interdisciplinaires, plutôt que de séparer l'enseignement de la langue de la recherche consacrée à la culture. « Ce que l'expression 'études romanes' incarne, c'est la force même de nos équipes composées de chercheurs qui utilisent différentes approches — historique, linguistique, philologique, sociopolitique, culturelle, visuelle et théorique - dans l'enseignement et l'étude des langues, des textes aussi bien littéraires que non-littéraires et des cultures de l'Europe, de la Méditerranée et des Amériques », déclare Luisetti ${ }^{6}$.

Un tel changement peut certes aussi s'interpréter comme le résultat d'une conception inspirée du marketing et comme un désir (mais certains diront qu'il s'agit d'une nécessité) de se présenter aux potentiels étudiants/consommateurs sous un jour nouveau, plus en phase avec les goûts d'un public contemporain que la littérature, et la lecture en général, rebuterait de plus en plus : à l'âge de la culture numérique et de ce qu'on appelle désormais les digital humanities, le cinéma, la pop culture, la publicité, la bande dessinée, les mangas - l'image en général - prennent, dans ce modèle d'enseignement, une importance primordiale au point où la littérature se voit reléguer au deuxième plan. Et on aurait tort de sous-estimer aujourd'hui l'importance que prend le nom dans le choix d'un cursus ou même d'un cours de la part d'un étudiant américain dont l'objectif de plus en plus affiché, une fois diplômé, sera bien sûr de rentabiliser son investissement en trouvant le poste auquel l'université doit forcément le préparer. Il ne fait aucun doute que cet étudiant de 2018 sera plus sensible à un intitulé du type «French Cultural Masterpieces » que «French Literature » et qu'il aura tendance à privilégier le premier plutôt que le second même si le contenu est plus ou moins le même dans les deux cours. En 2014, la directrice du Department of Romance Languages and Literatures de l'université d'Harvard n'hésitait pas à admettre que sa faculté « explorait différentes approches, comme une meilleure communication auprès des étudiants concernant la valeur des cours, la présentation de davantage de cours pour les non-spécialistes de façon à faire en sorte que la faculté apparaisse

${ }^{6}$ « This shift follows a national trend of other departments of Romance studies - at institutions such as Cornell and Duke universities - that have attempted to break down traditional methodological and national barriers to emphasize interdisciplinary research and scholarship, rather than separating language instruction and cultural research. "What 'Romance studies' captures is a key strength of our faculty, composed of scholars who apply a variety of approaches - historical, linguistic, philological, sociopolitical, cultural, visual and theoretical - to the teaching and study of languages and literary and nonliterary texts and cultures of Europe, the Mediterranean and the Americas," said Luisetti. » (A New Name for 106-year-old Department: Romance Studies, <http:// global.unc.edu/news/a-new-name-for-106-year-old-department-romance-studies/>). Toutes les traductions sont les miennes. 
plus pertinente aux étudiants » ajoutant également que la même faculté « envisageait de modifier le nom de son programme de premier cycle intitulé jusqu'alors Romance Languages and Literatures ${ }^{7}$. Mais au-delà de l'intitulé d'un cours, c'est aussi et surtout son contenu et les aspects pratiques de ce cours, et finalement les retombées économiques en découlant pourraient-on dire, qui retiennent avant tout l'attention des étudiants de $2018^{8}$ : « Les étudiants veulent connaître les avantages pratiques de leurs cours ", déclare la responsable des études de premier cycle du Department of Romance Languages and Literatures de l'université d'Harvard. «Les étudiants ne savent pas forcément ce qu'impliquent les études de langues et littératures romanes. Qu'est-ce qu'on fait avec ça ?»" .

Il y a déjà plusieurs années que les universités américaines, leurs étudiants et leur personnel, tant administratif qu'enseignant, empruntent au monde de l'entreprise et du marketing des concepts qui ont soi-disant fait leurs preuves. En cela aussi, l'université américaine, étant donné ses impératifs financiers grandissants et sa dépendance extrême vis-à-vis des inscriptions et des renouvellements d'inscriptions une année sur l'autre, se rapproche de plus en plus de l'univers de l'Amérique des Affaires (le célèbre Corporate America) où seule une croissance solide et régulière permet de générer des profits et de dégager des marges de manœuvre. Certains commentateurs ont commencé à parler de corporate neo-liberal universities dès le début des années $90^{10}$. L'importance qu'a prise ces dernières ce que l'on appelle dans le jargon universitaire américain la retention (le maintien, ou la fidélisation pourrait-on dire, des étudiants dans l'université d'une année sur l'autre) en dit long sur les modèles que privilégient de plus en plus les équipes administratives des universités aussi bien privées que publiques. Un des travers parmi les plus pervers de ce type de politique s'observe dans le fait que le haut personnel administratif est de moins en moins recruté parmi le corps enseignant, comme c'est encore le cas en Europe, mais de plus en plus au contraire au sein d'entreprises, de fondations publiques et privées, des corps gouvernementaux avec tous les avantages que cela représente pour les personnes en question (dont des salaires qui dépassent largement le million de dollars pour certains présidents

7 J.E. Steinman, «Amid Shrinking Enrollment, Romance Languages and Literatures Plans To Redesign Curriculum », The Harvard Crimson, 26 mars 2014 (je souligne), <http://www.thecrimson.com/article/2014/3/26/romance-languages-curriculum-change/>.

${ }^{8}$ Sept diplômés américains sur dix sortent des universités publiques avec des dettes contractées à cause de prêts étudiants constituant un marché juteux pour les banques et les instituts de crédit. La moyenne nationale de cette dette en 2017 est de $\$ 26,700$ par étudiant endetté. En règle générale, ces dettes doivent être remboursées par les étudiants à partir d'une année après l'obtention du diplôme, ce qui force nombre d'entre eux à accepter des offres d'emploi parfois en deçà de leurs ambitions.

9 J.E. Steinman, op. cit.

${ }^{10}$ Voir S. Slaughter et G. Rhoades, «The Neo-Liberal University », New Labor Forum 6 (printemps-été 2000), pp. 73-79. 
d'université, y compris dans le secteur public ${ }^{11}$ ) et tous les inconvénients qu'on imagine pour le reste du personnel universitaire, depuis le concierge jusqu'au professeur en passant par le petit personnel administratif, condamné à voir ses revenus stagner quand ils ne sont pas rognés comme cela a été le cas bien avant la crise des prêts à risque dans de nombreuses universités américaines : en 1993, par exemple, il était déjà question que les enseignants et le personnel des vingt universités du système public de l'enseignement supérieur de Californie subissent une baisse de salaire de l'ordre de $10 \%$ afin de rééquilibrer les comptes de l'état et d'éviter la suppression de plusieurs programmes et le licenciement de certaines catégories du personnel ${ }^{12}$. En 2015, un journaliste du New York Times indiquait, chiffres du Department of Education à l'appui, que le nombre de postes administratifs dans les universités américaines avait augmenté de $60 \%$ en une quinzaine d'années, de 1993 à 2009. Une autre étude, reprise par le même article, révélait également que le nombre de postes dans l'administration du système public de l'enseignement supérieur de la Californie était passé de 3800 à 12183 en un peu plus de trente ans, de 1975 à 2008, soit une croissance à peine croyable de l'ordre de plus de $220 \%$ alors que le nombre d'enseignants avait stagné sur la même période. Comme on le constate et quoi qu'il arrive, il semblerait qu'on en revienne sans cesse à l'économie et à l'argent lorsqu'il s'agit de tracer un état des lieux de l'université américaine contemporaine ${ }^{13}$. Mais qu'en est-il des facultés de langues et littératures romanes?

${ }^{11}$ En 2017, The Chronicle of Higher Education révélait que, sur « 250 high officials at public university systems, taking into account base pay plus bonus pay and other compensation, the highest paid public university official was President Michael Crow of Arizona State [University], who made an astounding $\$ 1,554,058$. William H. McRaven of the University of Texas system and Michael K. Young of Texas A\&M earned the highest base salaries public universities, McRaven getting \$1.2 million and Young \$1 million. According to the data, public university leaders were paid on average $\$ 464,000$ last calendar year » (L. Sonnenberg, « The Highest-Paid Public University Presidents », Forbes, 17 juillet 2017, <https://www.forbes.com/sites/laurensonnenberg/2017/07/17/ the-top-paid-public-university-presidents/\#5b387d53114c >).

${ }^{12}$ L. Gordon, « 10\% Pay Cut Urged for Cal State Faculty, Staff : Education: Salary reductions would avert layoffs and program eliminations, San Diego State president says », The Los Angeles Times, 20 mai 1992, <http://articles.latimes.com/1992-05-20/news/mn-180_1_cal-state-fullerton>. Depuis de nombreuses années maintenant, des universités en Arizona, en Californie, en Illinois, au Kentucky (pour ne citer que ces états) ont parfois recours, avec l'assentiment des syndicats quand ces derniers existent encore, à des congés forcés non payés (furloughs) pour tous leurs employés afin d'assainir leurs finances. Pour les enseignants, cela consiste généralement à ne pas se présenter sur leur lieu de travail le vendredi, jour où peu d'enseignants donnent cours de toute façon étant donné que les étudiants américains ont souvent tendance à éviter de suivre des cours en fin de semaine. Pour un exposé complet des conséquences de la crise des prêts à risque, voir J.R. Brown et C.M. Hoxby (dir.), How the Financial Crisis and Great Recession Affected Higher Education, University of Chicago Press, Chicago 2015.

${ }^{13}$ P. Campos, « The Real Reason College Tuition Costs So Much », New York Times Sunday Review, 4 avril 2015, <https://www.nytimes.com/2015/04/05/opinion/sunday/the-real-reason-collegetuition-costs-so-much.html? $\mathrm{r}=0 />$. 
La chute de l'URSS et de son modèle économique et social et la fin de la Guerre Froide ainsi que l'émergence de nouvelles puissances économiques, en Asie et au Moyen-Orient notamment, constituent sans doute un autre exemple de bouleversement géopolitique ayant eu (et continuant à avoir) des répercussions importantes sur l'évolution de l'enseignement des langues étrangères aux États-Unis et la désaffection de certaines d'entre elles comme le russe, par exemple (et dans une moindre mesure, d'autres langues européennes telles que l'allemand), qui avait bénéficié du climat de la guerre froide depuis les années 50 en faveur aujourd'hui des langues et cultures chinoise et arabe. La crise de 2007 a fortement accentué ce mouvement forçant les états américains (et les mécènes et autres donateurs) à réduire, parfois de façon drastique ${ }^{14}$, leurs dotations annuelles aux universités et forçant ainsi ces dernières à se tourner rapidement et de plus en plus régulièrement, pour rééquilibrer leurs budgets, vers des sources de financement alternatives dont certaines, parmi les plus lucratives, se situent en Asie et au Moyen-Orient où le développement économique générait encore, jusqu'à récemment, des surplus ayant disparu pratiquement du jour au lendemain aux États-Unis avec la crise des prêts à risque. C'est ainsi qu'on a vu arriver une nouvelle population étudiante composée principalement de citoyens chinois et originaires des pays du Golfe ${ }^{15}$, population baptisée par les médias de 'Génération Parachute', dont les parents, ayant atteint un niveau de vie élevé dans leur pays d'origine, souhaitent qu'ils complètent leurs études au sein d'une université américaine, considérées par d'aucuns comme une source de prestige $^{16}$. Comme on peut l'imaginer, l'émergence de nouvelles richesses suscite bien des convoitises de la part des universités américaines à la recherche désespérée de

${ }^{14}$ Entre les années universitaires 2007-2008 et 2015-2016, vingt-six états (sur cinquante) ont réduit le budget de leurs universités publiques de $20 \%$, neuf autres états l'ont réduit de $30 \%$. Les états d'Arizona et de l'Illinois ont, quant à eux, réduit leurs budgets respectifs de la moitié de ce qu'ils étaient avant la crise. Voir M. Mitchell, M. Leachman, K. Masterson, « Funding Down, Tuition Up State Cuts to Higher Education Threaten Quality and Affordability at Public Colleges », Center on Budget and Policy Priorities, <https://www.cbpp.org/research/state-budget-and-tax/funding-down-tuition-up/>.

15 En 2015, par exemple, le programme de bourse portant le nom du roi Abdullah, s'élevant à six milliards de dollars, a permis de financer en moyenne $90 \%$ des frais d'inscription, de couverture santé, de transport et parfois même les salaires de très nombreux étudiants saoudiens étudiant aux États-Unis. Voir L. Walcutt, «The Scholarship Struggle Saudi Arabian Students Are Facing », Forbes, 28 septembre 2016, <https://www.forbes.com/sites/leifwalcutt/2016/09/28/the-scholarship-struggle-saudi-arabianstudents-are-facing/\#706ea8931cd9/>. La politique du Président Donald Trump à l'égard des étrangers risque, bien entendu, de remettre en cause ce qui représente depuis plusieurs années une véritable manne pour de nombreuses universités américaines. Voir E. Deruy, « How Trump’s Immigration Order Is Affecting Higher Education », The Atlantic, 30 janvier 2017, <https:/www.theatlantic.com/education/ archive/2017/01/how-trumps-muslim-immigration-order-could-affect-higher-education/514925/>.

16 B. Larmer, "The Generation Parachute ", The New York Times, 2 février 2017, <https:// www.nytimes.com/2017/02/02/magazine/the-parachute-generation.html>. Ce que certains experts voient (d'un mauvais œil) comme une nouvelle forme de dépendance financière de la part des universités américaines (les étudiants étrangers payent en effet des frais d'inscription plus élevés que les étudiants américains et génèrent donc davantage de revenus) n'est pas sans créer d'autres problèmes d'ordre culturel. Voir M. Schiavenza, « The Tenuous Relationship Between American 
sources de financement alternatives. Malheureusement, l'explosion, ces dernières années aux États-Unis, de l'étude des langues et cultures chinoise et arabe ${ }^{17}$ (bien souvent grâce à ces mêmes étudiants chinois et arabes qui, paradoxalement, s'inscrivent dans des cours enseignés par des chercheurs américains pour y étudier leur propre littérature et culture d'origine) a provoqué un déséquilibre et conduit à un affaiblissement des facultés de langues et cultures européennes, suivant une dynamique qui est celle des vases communicants.

La question cruciale de la rentabilité des universités, liée à une situation financière compromise, devenue incertaine de façon quasi systémique, et à l'augmentation considérable des frais d'inscription pour contrebalancer la diminution des dotations des états aux universités publiques, ont eu d'autres effets négatifs sur l'évolution de l'enseignement des langues et littératures étrangères. Suivant une logique de rentabilisation, l'administration des universités fait de plus en plus pression sur les facultés pour remplir les classes afin de réduire les effectifs du corps professoral, négligeant cette spécificité de l'enseignement des langues étrangères qui veut que - et toutes les études le démontrent amplement - les résultats soient proportionnels au nombre d'étudiants dans une même classe : un cours magistral devant une centaine d'étudiants n'a jamais permis à ces derniers de faire des progrès significatifs sur le plan linguistique. On constate depuis plusieurs années que les départs à la retraite d'enseignants ne donnent plus automatiquement lieu à des remplacements comme c'était le cas par le passé. Les facultés doivent de plus en plus faire appel, en cas de besoin urgent (en début d'année universitaire généralement), à un personnel qui, s'il est disponible pour quelques semaines ou plusieurs mois, n'est pas toujours de la meilleure qualité, généralement mal payé et souvent sans couverture santé. Lorsque, une fois n'est pas coutume, les départs à la retraite donnent lieu à des remplacements, l'administration aura désormais tendance à favoriser la création de postes au profil transversal, désignés par les expressions « interdisciplinaire » ou « multidisciplinaire ». L'embauche de jeunes enseignants/chercheurs se fait donc de plus en plus selon ce modèle transversal entre les schools et les facultés d'une même université (et parfois même sur plusieurs campus à la fois) mettant en péril l'équilibre des programmes académiques qui doivent tailler chaque année un peu plus dans leur liste de cours et se contenter d'assurer l'essentiel. Le corollaire de ce type de recrutement en phase de généralisation est bien sûr que l'enseignement dans la langue étrangère (target language) se raréfie sous la pression de la rentabilisation et du « remplissage » des classes, l'anglais devenant la lingua franca de l'enseignement y compris dans les

Universities and Chinese Students », The Atlantic, 30 mai 2015, <https:/www.theatlantic.com/ education/archive/2015/05/american-universities-are-addicted-to-chinese-students/394517/>.

${ }^{17}$ Selon le Pew Research Center, l'augmentation de 2000 à 2014 du taux de croissance de l'étude de l'arabe aux États-Unis a été de $29 \%$. Un chiffre qui fait rêver les facultés de langues romanes... 
facultés de langue étrangère et les programmes de master et de doctorat de manière à attirer un public plus nombreux. Un cours enseigné en langue étrangère représente désormais un obstacle linguistique - et donc financier - dont de nombreuses universités aimeraient bien se débarrasser une fois pour toute. Maintenir et continuer à développer un haut niveau de maîtrise des langues étrangères parmi les étudiants américains devient dès lors un défi de plus en plus difficile à relever pour les enseignants de l'université dont de nombreux étudiants ont chaque année moins d'opportunités de suivre des cours dans la langue d'origine. Les premiers à pâtir de cette situation sont bien sûr les écoles primaires, collèges et lycées, qui par conséquent rencontrent des difficultés à recruter un personnel hautement qualifié et maîtrisant bien la langue pour enseigner les langues étrangères aux élèves de ces classes qui fourniront plus tard aux universités le plus gros contingent d'étudiants. Or c'est au niveau des collèges et des lycées, et même des écoles primaires, que le combat du recrutement doit être engagé car ce sont ces classes qui offriront aux universités de futurs étudiants. Mais pour enseigner les langues étrangères dans les établissements secondaires encore faut-il avoir à disposition des enseignants bien préparés. Comme on le constate, le serpent issu de la révolution néo-libérale des universités américaines se mord la queue.

La réduction et l'évolution de l'offre sur le marché du travail depuis la crise des prêts à risque a fortement accentué un mouvement qui avait été initié par le boom technologique des années 90 . Ce mouvement a eu lui aussi des conséquences contrastées sur l'étude des langues et littératures européennes au sein des universités américaines. Certes, la généralisation des supports numériques et le développement des cours hybrides et/ou exclusivement sur internet a permis aux facultés de langues et de littératures de proposer d'autres cours et d'attirer et de fidéliser un public différent (notamment des mères/pères de famille travaillant à la maison ou des personnes se trouvant déjà dans la vie active) mais ce phénomène se développe bien souvent au détriment du corps enseignant encore une fois puisque le personnel engagé pour enseigner ce type de cours en ligne est lui aussi bien souvent précaire, mal payé, sans couverture santé ni droits à la retraite, ce qui rend plus compliqué encore le recrutement et le maintien dans le temps de cette nouvelle catégorie de personnel enseignant somme toute très volatile. Autant dire que le prestige et l'utilité de la littérature, dans un tel environnement, où la priorité est la recherche d'un emploi de plus en plus difficile à trouver et à conserver et nécessitant souvent plus de compétences techniques, en viennent forcément à être remises en cause par l'administration des universités, forcées, devant leur dépendance toujours plus accrue vis-à-vis des frais d'inscription, à revoir leurs stratégies pour les adapter aux lois de l'offre et de la demande. Aussi un enseignant se souciant naturellement du futur professionnel de ses étudiants éprouvera-t-il de plus en plus de scrupules à encourager ces derniers à se spécialiser en langues et en littératures romanes étant donné l'état réel de l'économie américaine et du marché du travail, les postes d'enseignants dans des conditions d'embauche 
décentes se faisant de plus en plus rares. Le système de la double spécialisation (double major) peut fournir une solution partielle pour maintenir, à défaut de développer, l'enseignement des langues parmi une population qui, bien souvent il faut bien l'avouer, ne voit pas l'utilité de parler sur un territoire aussi grand que le sont les États-Unis une autre langue que l'anglais ou l'espagnol. L'avantage de la double spécialisation est qu'elle permet à tout étudiant en ayant les capacités de se spécialiser dans deux disciplines différentes dès les quatre premières années de sa scolarité, ce qui est le cas de nombreux étudiants de langues étrangères. Ainsi, par exemple, un étudiant ayant combiné l'étude du droit international et du français aura-t-il plus de débouchés professionnels que s'il avait étudié uniquement le français comme matière principale. Telles un serpent qui se mord la queue encore une fois, les langues et littératures étrangères semblent de plus en plus destinées à devenir, dans le contexte universitaire d'une économie libérale généralisée et globale, ce que l'administration de ces mêmes universités nomment des «service departments », c'est-à-dire des enseignements d'appoint et non plus des filières en elles-mêmes avec des postes à la clé au sortir de l'université pour les étudiants et des possibilités de croissance constantes pour les facultés et leurs enseignants. Quel sera le rôle de ces matières et de leurs enseignants/chercheurs dans les années à venir ? L'évolution de la situation économique dans le monde pourra-t-elle fournir des moyens financiers et donc matériels à ces programmes pour qu'ils se réinventent et s'assurent une place légitime au sein de l'université néo-libérale et de la société du futur ? Quelle sera cette place ? Ce sont certaines des questions qui se posent de façon inquiétante depuis plusieurs années et auxquelles les universités américaines n'ont jusqu'ici tenté de répondre qu'au coup par coup, sans réel effort de concertation au niveau national étant donné l'absence d'un système fédéral pour ce qui concerne l'enseignement supérieur, à défaut de pouvoir engager des réformes qui permettraient de régler de façon plus pérenne le problème récurrent du financement.

Si l'on en croit l'adage selon lequel les États-Unis sont souvent en avance sur le reste du monde, faut-il donc penser que la situation des universités américaines aujourd'hui préfigure celle des universités européennes de demain ? ${ }^{18}$ On ne le souhaite pas et on a même du mal à le croire étant donné la structure et les modes de financement des universités dans la plupart des pays de l'Union européenne.

${ }^{18}$ Contrairement aux universités du reste de l'Europe, les universités britanniques semblent se rapprocher de plus en plus de leurs consœurs de l'autre côté de l'Atlantique y compris, comme le suggère André Spicer, pour ce qui concerne les problèmes rencontrés par ces dernières : « $\mathrm{Au}$ Royaume-Uni, deux tiers des universités ont désormais plus de personnel administratif que d'enseignants. Un expert de l'enseignement supérieur prédit la naissance du tout administratif à l'université » (A. Spicer, « Universities are broke. So let's cut the pointless admin and get back to teaching ", The Guardian, 21 août 2017, <https:/www.theguardian.com/commentisfree/2017/aug/21/ universities-broke-cut-pointless-admin-teaching? $\mathrm{CMP}=$ share btn $\mathrm{fb} />$ ). 
Les Européens semblent aussi attachés à leur système éducatif et prêts à des sacrifices pour le préserver. La force et les répercussions de la polémique provoquée en 2009, par exemple, par les propos de l'ancien Président de la République française Nicolas Sarkozy, au sujet de La Princesse de Clèves ont révélé combien le patrimoine littéraire conservait en France (mais aussi dans le reste de l'Europe), en dépit des évolutions politiques, sociales, technologiques, culturelles, etc. de ces trente dernières années, une dimension existentielle, touchant au cœur même de l'identité culturelle et spirituelle de la nation et de son peuple. On peut toujours penser, comme le faisait Ernst-Robert Curtius en 1930, à une forme d'exceptionnalisme que les Français aiment eux-mêmes à cultiver un peu trop :

La littérature, écrivait Curtius, joue un rôle capital dans la conscience que la France prend d'ellemême et de sa civilisation. Aucune autre nation ne lui accorde une place comparable. Il n'y a qu'en France où la nation entière considère la littérature comme l'expression représentative de ses destinées ${ }^{19}$.

En 2018, les propos du philologue allemand me semblent définir tout autant la France que la plupart des autres nations européennes, y compris la Russie, et c'est sans doute ce qui différencie le plus les États-Unis de certains pays de l'Union européenne ou, pour reprendre la sinistre formule de George W. Bush, de la « vieille Europe ». S'il ne prédit en rien l'avenir de l'Europe, le monde universitaire américain, en tant que miroir de l'évolution des mentalités et des pratiques sociales américaines depuis la révolution néo-libérale et la chute du mur de Berlin, peut tout au moins fournir un cadre représentatif et révélateur de la façon dont cette évolution s'est manifestée concrètement dans les faits, en particulier si l'on considère plus précisément, comme nous avons essayé de le faire dans cet essai lorsque cela était possible, la vie des facultés de langues romanes au sein des universités publiques et privées américaines.

\section{LANGUAGES AND LITERATURES OF 'OLD EUROPE' IN AMERICAN UNIVERSITIES: AN ASSESSMENT}

\section{Summary}

Having completed his literature studies in France, the author of this essay started teaching in the United States in 1988 in both private and public universities. This essay is the result of his observations made as a teacher, a scholar and an administrator on the evolution and the shifts occurred in departments of romance languages these past thirty years. Since the 1980 s, neo-liberal politics and repetitive economic crisis encouraged states to drastically reduce their financial support to public universities forcing them to turn to other forms of financing including juicy contracts with Asian and the Middle Eastern countries where economic development generates surplus. This essay studies the

${ }^{19}$ E.-R. Curtius, Essai sur la France, traduit par Jacques Benoîst-Méchin, Grasset, Paris 1941 [1930], p. 145. 
consequences on departments of Romance languages of a university policy conducted in favor of the development of Chinese and Arabic languages, as well as sciences instead of European languages and the humanities in general, and shows how the preference given to those newly developing languages has weaken departments of European studies as a result.

Key words: teaching, romance languages, United States, American universities, neoliberal university. 